\title{
Relação entre a cobertura de atenção básica das capitais do Nordeste e taxas de internações por causas sensíveis à saúde
}

\author{
Relatioship between primary care coverage of northeaste capitals and health-sensitive \\ hospitalizatioon rates
}

\author{
Eduarda de Brito Pereira ${ }^{1}$, Paloma Santos da Hora ${ }^{1}$, Aline Silva Rocha ${ }^{1}$, Samilly Silva Miranda ${ }^{2 *}$ \\ ${ }^{1}$ Graduanda no Bacharelado Interdisciplinar em Saúde; ${ }^{2}$ Doutora em Saúde Coletiva pela Universidade Estadual de \\ Feira de Santana.
}

\begin{abstract}
Resumo
Introdução: as Doenças Crônicas Não Transmissíveis (DCNTs) correspondem a um grave problema de saúde pública e representam um empecilho frente ao desenvolvimento humano. Objetivou-se apresentar a distribuição da cobertura da atenção da básica no período de 2004 a 2009 e descrever as taxas de internações para causas sensíveis em saúde e doenças hipertensivas nas capitais dos estados da região Nordeste do Brasil. Metodologia: realizou-se estudo ecológico, a partir de dados secundários disponíveis no Departamento de Informática do Sistema Único de Saúde (DATASUS) e do Sistema de Informações Hospitalares do SUS (SIHSUS). Os dados foram exportados dos sistemas de informação e posteriormente foram obtidas as medidas de frequências relativas. Os dados foram tabulados e apresentados em formato de gráficos. Resultados: os resultados sobre a distribuição da cobertura de atenção básica revelaram que Aracaju e Teresina são as capitais com maior cobertura (95,3\% e 90,0\%, respectivamente); nas taxas de internações sensíveis à atenção básica, João Pessoa e Teresina aparecem com as maiores taxas, 147,2 e 155,2, respectivamente, no ano de 2005; no que se relaciona às taxas de internações por doenças hipertensivas, Teresina foi a capital com as maiores taxas de 2004 a 2009 (15,5\% e 12,3\%, respectivamente), mesmo com a diminuição ocorrida a partir de 2006. Conclusão: de acordo com os resultados encontrados as capitais que apresentavam maior cobertura de atenção básica, são aquelas que apresentaram as maiores taxas de internações por causas sensíveis. Como a análise foi descritiva, não foi possível mensurar a associação entre os eventos, nem questões relacionadas à qualidade e efetividade da atenção prestada. Dessa forma, sugere-se que sejam realizados estudos analíticos sobre a avaliação da efetividade da atenção básica no Nordeste.
\end{abstract}

Palavras-chave: Atenção primária. Internação hospitalar. Pressão arterial.

\begin{abstract}
Introduction: uncommunicable Chronic Diseases (DCNT, for its acronym in Portuguese) constitutes a serious public health problem and represents an obstacle to the human development. The objective was to present paper the distribution of coverage of primary care and describe hospital admission rate for sensitive health conditions and hypertensive diseases and primary care coverage in capital states of North-eastern Brazil during the period of 2004-2009. Methodology: an ecological study was conducted from the information available in the Department of Informatics of the Unified Health System (DATASUS, for its acronym in Portuguese) and Hospitals Information System of Unified Health System (SIHSUS, for its acronym in Portuguese) databases. The data were exported from the information systems and subsequently the relative frequency measurements were obtained. The data was formatted and presented in graphs. Results: the results on the distribution of primary care, revealed that Aracaju and Teresina are the cities with the highest coverage (95.3\% and $90.0 \%$, respectively). Regarding the primary care admission rates during the year of 2005, João Pessoa and Teresina stand out with the highest rates, 147,2 and 155,2 respectively. When it comes to admissions rates for hypertensive diseases, Teresina was the capital with highest rates from 2004 to 2009 (15.5\% and 12.3\%, respectively), despite the decreasing since 2006. Conclusion: according to the results founded, the capitals that had the highest coverage of primary care are those which had the highest rates of hospitalizations for sensitive causes. As the analysis was descriptive, it was not possible to measure the association between the events, nor questions related to the quality and effectiveness of the care provided. Thus, it is suggested that analytical studies be carried out on the evaluation of the effectiveness of primary care in the northeast of the country.
\end{abstract}

Keywords: Primary care. Hospital admissions. Blood pressure.

\section{INTRODUÇÃO}

Com o advento da globalização, várias mudanças ocorreram no perfil epidemiológico da população e essas estão relacionadas direta ou indiretamente com o modo

Correspondente/Corresponding: *Samilly Silva Miranda - End: Instituto de Saúde Coletiva. Rua Basílio da Gama, s/n - Canela, Salvador - BA, CEP: 40110-040. - Tel: (71) 3283-7450-E-mail: samillymiranda@ufba.br de vida, costumes e hábitos das pessoas. Essas alterações favorecem o surgimento de novos riscos, novas vulnerabilidades e angústias que colaboram para a incidência e aumento da prevalência de doenças crônicas não transmissíveis (DCNT) (FERREIRA et al., 2009).

As Doenças Crônicas Não Transmissíveis, correspondem a um grave problema de saúde pública a nível global e representam um empecilho frente ao desenvolvimento 
humano (SCHMIDT, 2011). De acordo com a Organização Mundial de Saúde (OMS), 70\% dos óbitos ocorridos no mundo, até 2018, foram relacionados às DCNTs, representadas por quatro grupos de doenças - cardiovasculares, câncer, doença respiratória crônica e diabetes - como as principais responsáveis pela maior parte dos óbitos.

Como o Brasil é um país de dimensões continentais, mudanças no perfil epidemiológico da população não acontecem uniformemente, visto que ocorrem disparidades socioeconômicas e de acesso aos serviços de saúde nas regiões brasileiras. A partir dessa observação, Araújo (2012) intitula este modelo como um "modelo polarizado de transição", no qual as mudanças ocorrem de formas diferentes de acordo com a classificação do país: desenvolvido ou em desenvolvimento.

Historicamente, o Nordeste foi a região de maior concentração de pobrezas do país, gerando um enorme impacto na saúde da população residente nessa região, sobretudo, no que se refere às doenças infecciosas e evitáveis, refletindo diretamente no número de internações por causas sensíveis à atenção básica (ICSAB). Estas, se referem às condições de saúde que, se tratadas de forma adequada na atenção primária, evitariam internações (PINTO; GIOVANELLA, 2018). Dentro do grupo de 20 condições de saúde da ICSAB, encontra-se a hipertensão.

Definida como condição clínica multifatorial e identificada por níveis elevados e sustentados de pressão arterial, a Hipertensão Arterial Sistêmica (HAS) é uma DCNTs que se vincula a alterações funcionais dos órgãos alvos e alterações metabólicas (FERREIRA et al., 2009). A prevalência é de $30 \%$ para a população adulta brasileira e de acordo a pesquisa Sistema de Vigilância de Fatores de Risco e Proteção para Doenças Crônicas por Inquérito Telefônico (VIGITEL, 2018) 24,7\% dos moradores das capitais brasileiras são atingidos pela a hipertensão, sendo as capitais do Nordeste: Maceió $(27,1 \%)$; Recife $(26,5 \%)$ e João Pessoa $(26,1 \%)$ com maior prevalência.

Haja vista que a HAS acomete muitas pessoas gerando assim muitas vítimas fatais, dados do Sistema de Informações de Mortalidade (SIM), do Ministério da Saúde, mostram ainda que, em 2017, o Brasil registrou 141.878 mortes devido a hipertensão ou a causas relacionadas a ela. Santos, Prado e Santos (2018), no seu estudo sobre mortalidade por doenças hipertensivas no Brasil constatou que a taxa global de mortalidade nas regiões brasileiras, para cada 10.000 habitantes foi de: norte $(1,25)$; nordeste $(2,69)$; centro-oeste $(2,06)$; sudeste $(2,48)$ e sul $(2,04)$. É notável o elevado índice encontrado na região nordeste, no qual teve um total de 26.900 óbitos causados por HAS.

Diante do cenário apresentado, as doenças crônicas, neste caso a HAS, merecem atenção dos órgãos públicos, no sentido da compreensão de que o processo saúde-doença é socialmente determinado. Assim, em meio às várias propostas ofertadas para o controle da HAS, no Brasil, o destaque vai para as ações concentradas no trabalho da Estratégia de Saúde da Família (ESF). Além da ampliação do acesso aos serviços básicos de saúde, a ESF proporcionou a criação de vínculos entre profissionais e usuários (BRASIL. MINISTÉRIO DA SAÚDE, 1997).

A relevância em trabalhar com os dados da região Nordeste se justifica pelos números elevados de mortes que tem como causa básica a HAS, além de ser uma das mais vulnerabilizadas do país, o que tende a influenciar nas condições de saúde desta população. Assim, o objetivo deste trabalho é apresentar a distribuição da cobertura da atenção básica no período de 2004 a 2009 e descrever as taxas de internações para causas sensíveis em saúde e doenças hipertensivas nas capitais dos estados da região Nordeste do Brasil.

\section{METODOLOGIA}

Trata-se de um estudo ecológico, realizado nas capitais dos estados da região Nordeste, do período de 2004 a 2009. Foram utilizados dados secundários provenientes do banco de dados do Sistema de Informação Hospitalares do SUS (SIHSUS) e dos Cadernos de Informação de Saúde, disponíveis no Departamento de Informática do Sistema Único de Saúde (DATASUS).

A região Nordeste, uma das cinco grandes regiões do Brasil, possui nove estados: Alagoas, Bahia, Ceará, Maranhão, Paraíba, Pernambuco, Piauí, Rio Grande do Norte e Sergipe. De acordo com dados do último censo demográfico do Instituto Brasileiro de Geografia e Estatística (IBGE) a população para a região, em 2010, correspondia a 53.081.950 indivíduos.

No site do DATASUS as informações de morbidade e de assistência à saúde das capitais dos estados da referida região foram coletadas a partir do link "informações de saúde", considerando o período de 2004 a 2009.

Para os dados de morbidade seguiu-se: Indicadores de saúde e pactuações, indicadores e dados básicos, indicadores de morbidade, taxa de internações hospitalares por causas selecionadas. Selecionou-se: Linha (capital), Coluna (não ativa), Conteúdo (Taxa de internação por doenças hipertensivas, taxa de internações sensíveis à atenção básica, internação por doenças hipertensivas, internações sensíveis à atenção básica e população) e Período (2004 a 2009).

Para análise da cobertura da ESF foram selecionados: Indicadores de saúde e pactuações, indicadores municipais, Cadernos de informação em saúde, no mapa de cada estado da região Nordeste e ao selecionar o Estado foi escolhido o caderno de informação em saúde referente a capital de cada estado. No caderno de informação em saúde foi elegido o capítulo pertencente a atenção básica e neste continham as informações do período (2004 a 2009).

A busca foi realizada em dias diferentes pelas integrantes da equipe, dividindo-se as capitais entre as mesmas. Não foi necessário recorrer às buscas documentais nas capitais já que o intuito do estudo foi utilizar os dados presentes no DATASUS. Não foi necessário submeter o 
trabalho ao comitê de ética, pois o mesmo não envolve pesquisa direta com a população.

Os dados foram exportados dos sistemas de informação para o programa Microsoft Office Excel 2016, onde foram organizados. Posteriormente empregou-se $a$ análise descritiva, obtendo as medidas de frequências relativas. Estas foram apresentadas em formato de gráficos.

\section{RESULTADOS}

Os resultados sobre a distribuição da cobertura de atenção básica, revelaram que Aracaju e Teresina são as capitais com maior cobertura $(95,3 \%$ e $90,0 \%$, respectivamente), sendo que Aracaju apresentou uma leve queda entre 2007 e 2008, mas voltou a crescer a partir de 2008. João Pessoa se destaca pelo crescimento da cobertura, chegando a alcançar os números de Teresina em 2009. Salvador e Maceió são as capitais com a cobertura de atenção básica mais baixas no período estudado, onde Salvador, no período de 2005 e 2007, apresentou um leve aumento na sua cobertura passando de $30,3 \%$ para $32,2 \%$, entrando em declínio a partir de 2007 e chegando a $28,9 \%$ em 2009. Maceió passou por momentos de instabilidade e pouco avanço em sua cobertura passando de $23,8 \%$ em 2004 para 26,2\% em 2009 (Gráfico 1).

Gráfico 1 - Distribuição da cobertura de atenção básica de acordo com as capitais da Região Nordeste de 2004 - 2009.

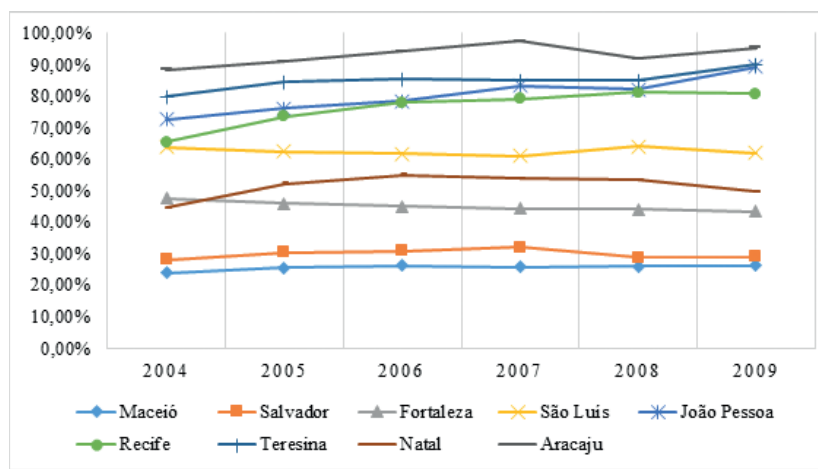

Fonte: Ministério da Saúde - Cadernos de Informação em Saúde/ DATASUS.

Nas taxas de internações sensíveis à atenção básica, João Pessoa e Teresina aparecem com as maiores taxas, 147,2 e 155,2, respectivamente, no ano de 2005, enquanto que Maceió tem declínio constante, saindo de 144,4 em 2004 para 52,8 em 2008. Fortaleza passa por uma oscilação, tendo a sua maior taxa no ano de 2004 , com 148,53 e atinge sua menor taxa no ano de 2008, chegando aos 104,9. Entretanto, Salvador e Aracaju têm as menores taxas: 53,8 e 86,2 em 2004 respectivamente, e ambas as capitais atingem a taxa de 44,0 no ano de 2009 (Gráfico 2).
Gráfico 2 - Taxas de internações sensíveis à atenção básica de acordo as capitais da Região Nordeste de 2004 - 2009.

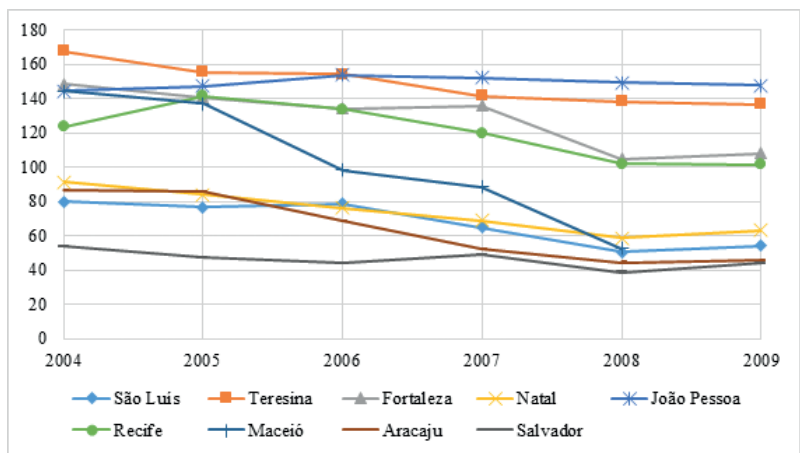

Fonte: Ministério da Saúde - Sistema de Informações Hospitalares do SUS (SIH/SUS) / Instituto Brasileiro de Geografia e Estatística (IBGE).

No que tange às taxas de internações por doenças hipertensivas, Teresina foi a capital com as maiores taxas de 2004 a 2009 (15,5\% e 12,3\%, respectivamente), mesmo com a diminuição ocorrida a partir de 2006. As outras capitais expressam números muito abaixo de Teresina, destacando-se Maceió e São Luís como as capitais com menores taxas de internações (1,1\% e 1,6\%). Ressalta-se ainda que, as taxas de Maceió demonstraram fases instáveis enquanto que São Luís evidencia um declínio acentuado entre 2004 e 2008 (Gráfico 3).

Gráfico 3 - Taxas de internações por doenças hipertensivas de acordo as capitais da Região Nordeste de 2004 - 2009.

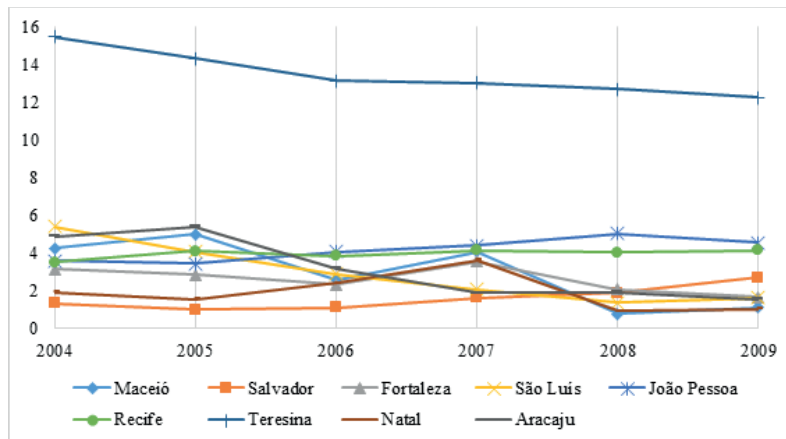

Fonte: Ministério da Saúde - Sistema de Informações Hospitalares do SUS (SIH/SUS) / Instituto Brasileiro de Geografia e Estatística (IBGE).

\section{DISCUSSÃO}

Diante da busca realizada, os resultados apontam que parcela considerável das capitais do Nordeste apresentam uma alta taxa de cobertura da atenção básica. Aquelas que possuem boa cobertura de atenção básica, têm elevadas taxas de internações por causas sensíveis, mas em relação às taxas de internação por doenças hipertensivas, a maioria das capitais se encontram com taxas baixas. E à medida que a taxa de cobertura diminuiu em algumas capitais, houve decréscimo também nas taxas de internações por causas sensíveis e hipertensão. 
Os resultados obtidos neste trabalho, corroboram com o estudo de Facchini et al. (2006), que sinalizou que durante o período de 1999 a 2004, a região Nordeste teve um crescimento relativo da cobertura da atenção básica maior do que no Sul do país, devido à expansão da ESF. Em relação às regiões com baixos índices de cobertura, a situação se relaciona com as circunstâncias históricas em que se desenvolveu o Sistema Único de Saúde (SUS) em cada capital, bem como aos problemas subsequentes ao processo de institucionalização do SUS no país, que não se desassocia da natureza do Estado e nem dos atributos da sociedade brasileira (PAIM; TEIXEIRA, 2007).

No caso específico de Salvador, capital com a segunda menor taxa de cobertura no período observado, os resultados foram semelhantes com a avaliação da cobertura de atenção básica feita no município, quando analisou os anos de 2000 - 2007 (CARDOSO; VIEIRA-DA-SILVA, 2012). Identificou-se como justificativa para os problemas relacionados a gestão municipal, principalmente, no que diz respeito à autonomia na administração dos recursos e inconstâncias no quadro dos dirigentes como explicação para a situação observada. Ademais, a correlação entre o aumento populacional frente ao crescimento insuficiente da oferta de serviços de saúde, gerou uma demanda assistencial além da suportada (CARDOSO; VIEIRA-DA-SILVA, 2012).

Em contrapartida ao município de Salvador, em Aracaju, capital de Sergipe, os gestores municipais encontraram mais autonomia para a administração dos recursos de saúde. De acordo Giovanella e colaboradores (2009), a decisão política da implantação da ESF como meio de fortalecimento da atenção básica no sistema municipal, é facilitado pelo legado institucional pois, Aracaju é habilitada pelas normas nacionais de operacionalização do SUS, como gestora plena do sistema de saúde.

Considerando a atenção básica enquanto ordenadora das Redes de Atenção à Saúde, o aumento das coberturas vistas na maioria das capitais, se deram através da implantação de Equipes de Saúde da Família. Através desta estratégia esperou-se ampliar o acesso e utilização da população a serviços de saúde, garantir longitudinalidade, integralidade, coordenação do cuidado, criação de vínculo, responsabilização, humanização e participação social. Esses atributos são essenciais para uma atenção básica resolutiva, com capacidade para promover melhores resultados em indicadores de saúde, maior satisfação dos usuários, maior equidade em saúde, além de menores custos para o Sistema de Saúde (BAHIA, 2015).

Os indicadores de internações por causas sensíveis à saúde, vêm sendo utilizados em diversos países como meios para medir a qualidade da atenção à saúde (ELIAS; MAGAJEWSKI, 2008). Na Espanha, as pesquisas têm procurado validar uma relação de causas de internações Hospitalares Sensíveis à Atenção Primária para medir a efetividade da Atenção Primária à Saúde, por entender que estas podem variar de acordo com os serviços de saúde oferecidos neste nível de atenção (BERMÚDEZ et al., 2004; CALDERÓN, 2003). Neste sentido, os resultados em relação às taxas de internações por causas sensíveis à saúde no Nordeste, também são condizentes com a literatura. No período selecionado para a análise neste artigo, Teresina e João Pessoa, mesmo sendo as capitais com altas taxas de cobertura da atenção básica, foram as capitais com as maiores taxas de internações por causa sensíveis, o que pode sinalizar uma baixa resolutividade nessas capitais.

O SUS vem experimentando mudanças na sua estrutura. Desde 1994, o Programa de Saúde da Família, ampliado posteriormente para a ESF, a priori, foi desenvolvido para melhorar o acesso à $A B S$ e a qualidade desta em todo o território. Seu aspecto estruturante dos sistemas municipais de saúde, visa à reorganização do modelo de atenção no SUS, coerente com os princípios da universalidade, equidade e integralidade (BRASIL, 2000). Neste sentido, para que os objetivos da ESF sejam atingidos, a atuação do Agente Comunitário de Saúde (ACS) é imprescindível pois, propicia a criação de vínculos entre a comunidade e a equipe. De acordo com o Caderno de Informações de Saúde das capitais do Piauí e da Paraíba, à medida que houve um aumento na cobertura da atenção básica da população, houve também um decréscimo na quantidade da população coberta pelo Programa de Agentes Comunitários de Saúde (PACS). Assim, considerando o papel fundamental do ACS para a ESF, as elevadas taxas de ICSAB observadas em Teresina e João Pessoa entre 2004-2009 se podem estar relacionadas também com a diminuição do número de ACS, contribuindo para a insipiência da ESF em doenças evitáveis.

Ainda, é importante sinalizar que o trabalho da equipe multiprofissional e interdisciplinar da ESF aos portadores de HAS visa à efetivação da integralidade em saúde no sentido de possibilitar uma atuação em saúde mais contextualizada, resolutiva na perspectiva da corresponsabilização de trabalhadores, usuários e suas famílias (CAMARGO; ANJOS; AMARAL, 2013). Dessa maneira, os resultados sobre internações por doenças hipertensivas na região Nordeste, onde Teresina aparece com alta taxa de internações, apesar da alta cobertura da atenção básica, acabam sendo reflexos da efetividade da ABS. Um estudo realizado na 16a Coordenadoria Regional de Saúde do Rio Grande do Sul (RAUPP et al., 2015), analisou trajetórias assistenciais de usuários vinculados à uma equipe de ESF, sugerindo baixa efetividade da ABS, tanto na prevenção quanto no diagnóstico das DCNT, pois muitos usuários foram diagnosticados após acessarem os níveis secundários e terciários. Os resultados deste estudo reforçam que a ESF só é efetiva quando as práticas não repetem o modelo biologicista e as equipes estabelecem vínculo com os usuários, variando do modo de implantação em cada município.

Entretanto, é importante salientar que condições socioeconômicas, nível de escolaridade, hábitos de vida, região, idade sexo e comportamento de procura por cuidados de saúde, são variáveis que influenciam nas taxas 
de internações e que não são passíveis de resolutividade na $A B S$, como apontam alguns estudos que demonstram que em regiões com baixo nível de escolaridade, as taxas de internações são maiores, comparadas às regiões mais desenvolvidas (REZENDE, 2010).

As limitações deste estudo estão atreladas ao fato dessa pesquisa ter sido realizada com dados secundários, bem como a possibilidade de haver subnotificações no que se refere às internações. Outro limitante concerne aos sistemas de informações em saúde utilizados, uma vez que esses apresentam problemas técnicos que dificultaram o levantamento dos dados.

\section{CONCLUSÃO}

De acordo com os resultados encontrados as capitais que apresentavam maior cobertura de atenção básica, são aquelas que apresentaram as maiores taxas de internações por causas sensíveis. Como esta investigação tem caráter apenas descritivo, não foi possível mensurar se existe associação entre estas questões. Ademais, os dados apresentados não demonstram questões relacionadas à qualidade e efetividade da atenção prestada, o que poderia estar associada ao aumento ou redução das internações. Dessa forma, sugere-se que sejam realizados estudos analíticos sobre a avaliação da efetividade da atenção básica no Nordeste, visto que, o mesmo, poderia contribuir para o planejamento de ações que reduzissem as internações por causas sensíveis e DCNTs, a exemplo da HAS.

\section{REFERÊNCIAS}

ARAÚJO, J. D. Polarização epidemiológica no Brasil. Epidemiol. Serv. saúde, Brasília, v.21, n.4, dez. 2012.

BAHIA. Pactuação das diretrizes, objetivos e indicadores 2013-2015 (SISPACTO) e programação das ações do sistema nacional de vigilância em saúde (PROGVS). Manual de orientação.2015.Disponível em:http:// www5.saude.ba.gov.br/obr/arquivos_download/MANUAL_SISPACTO_ ONLINE.pdf. Acesso dia: 01 de julho de 2019.

BERMÚDEZ, T. C. et al. Características organizativas de la atención primaria y hospitalización por los principals ambulatory care sensitive conditions. Aten. prim., Barcelona, v.33, n..6, p. 305-311, 2004.

BRASIL. Ministério da Saúde. Secretaria de Assistência à Saúde. Coordenação de Saúde da Comunidade. Saúde da família: uma estratégia para a reorientação do modelo assistencial. Brasília: Ministério da Saúde, 1997.

BRASIL. Ministério da Saúde. A implantação da Unidade de Saúde da Família. Brasília: Ministério da Saúde, 2000. (Caderno de Atenção Básica: Programa de Saúde da Família, v. 1).

CALDERÓN, S. M. et al. Factores asociados a la hospitalización por procesos sensibles a cuidados ambulatorios en los municipios. Gac. sanit., Barcelona, v.17, n.5, p. 360-367, 2003.

CAMARGO, R. A. A.; ANJOS, F. R.; AMARAL, M. F. Estratégia de Saúde da Família nas ações primárias de saúde ao portador de hipertensão arterial sistêmica. REME rev. min enferm., Belo Horizonte, v.17, n.4, p.864-872, 2013

CARDOSO, M. O.; VIEIRA-DA - SILVA, L. M. Avaliação da cobertura da atenção básica à saúde em Salvador, Bahia, Brasil (2000 a 2007). Cad. Saúde Pública, Rio de Janeiro, v.28, n.7, jul. 2012.

ELIAS, E.; MAGAJEWSKI, F. Atenção Primária à Saúde no sul de Santa Catarina: análise das internações por condições sensíveis à atenção primária, no período 1999-2004. Rev. bras. epidemiol., São Paulo, v.11, n.4, p.633-647, 2008.

FACCHINI, L. A. et al. Desempenho do PSF no Sul e no Nordeste do Brasil: avaliação institucional e epidemiológica da Atenção Básica à Saúde. Ciênc. Saúde Colet., Rio de Janeiro, v.11, n.3, p.669-681, 2006.

FERREIRA, S.R.G. et al. Frequência de hipertensão arterial e fatores associados: Brasil, 2006. Rev. Saúde Pública, São Paulo, v. 43, n.2, p. 98-106, 2009.

GIOVANELLA, Ligia et al. Saúde da família: limites e formas de atendimento integral de atenção à saúde no Brasil. Ciênc. Saúde Colet., Rio de Janeiro, v.14, n.3, p.783-794,2009.

PAIM, J. S.; TEIXEIRA, Carmen Fontes. Configuração institucional e gestão do Sistema Único de Saúde: problemas e desafios. Ciênc. Saúde Colet., Rio de Janeiro, v.12, p.1819-1829, 2007.

PINTO, L. F.; GIOVANELLA, L. Do Programa à Estratégia Saúde da Família: expansão do acesso e redução das internações por condições sensíveis à atenção básica (ICSAB). Ciênc. Saúde Colet., Rio de Janeiro, v.23, n.6, p.1903-1914, 2018.

RAUPP, L. M. et al. Doenças crônicas e trajetórias assistenciais: avaliação do sistema de saúde pequenos municípios. Physis, Rio de Janeiro, v. 25, n. 2, p. 615-634, 2015.

REZENDE, V. A. A resolutividade da atenção básica: uma revisão de literatura. Universidade Federal de Minas Gerais. 2010. 44f. Monografia (Especialização em Atenção Básica em Saúde da Família) - Faculdade de Medicina, Núcleo de Educação em Saúde Coletiva, Belo Horizonte, 2010.

SCHMIDT, M. I. et al. Doenças crônicas não transmissíveis no Brasil: sobrecarga e desafios atuais. Lancet, London, v. 377, n. 9781, 2011.

SANTOS, M.A.A.; PRADO, B.S.; SANTOS, D.M.S. Análise espacial e tendências de mortalidade associada a doenças hipertensivas nos estados e regiões do Brasil entre 2010 e 2014. Int. J. Cardiovasc. Sci., Rio de Janeiro, v. 31, n. 3, p. 250-257, 2018.

VIGITEL. Ministério da Saúde. Secretaria de Vigilância em Saúde Brasil. Secretaria de Vigilância em Saúde. Vigilância de fatores de risco e proteção para doenças crônicas por inquérito telefônico. Brasília: Ministério da Saúde, 2018. 132 p.

WORLD HEALTH ORGANIZATION (WHO). Hora de entregar: relatório da Comissão independente de alto nível da OMS sobre doenças não transmissíveis. Disponível em: https://www.who.int/ncds/management/ time-to-deliver/en/. Acesso em: 05 maio 2019.

Submetido em: 05/07/2019

Aceito em: 08/04/2020 